Pacific Journal of Mathematics

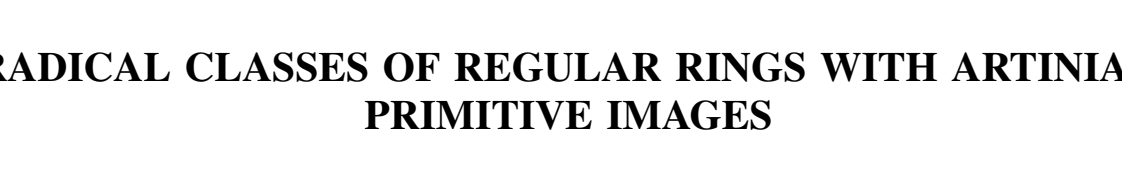




\title{
RADICAL CLASSES OF REGULAR RINGS WITH ARTINIAN PRIMITIVE IMAGES
}

\author{
B. J. GARDNER
}

This paper deals with radical classes consisting of regular rings, all of whose primitive homomorphic images are artinian (such rings will be called PA-regular). Noteworthy examples of such radical classes include, for each $n$, the class of regular rings satisfying the condition

$$
a \text { nilpotent } \Rightarrow a^{n}=0,
$$

and thus, in particular, the class of all strongly regular rings. It is shown that every radical class $\mathscr{R}$ consisting of PAregular rings is hereditary, and is the lower radical class defined by those of its members which are isomorphic to matrix rings of strongly regular rings with identities.

Moreover, for each $m$, the class of strongly regular rings for which the $m \times m$ matrix ring is in $\mathscr{R}$ is a radical class. This fact establishes a very important rôle for the radical classes of strongly regular rings, and accordingly a section is devoted to the latter. In the final section some attention is given to the question of closure of radical classes under direct products.

It is probably safe to say that more is known about the radical classes which contain all nilpotent rings than about those which contain none. Certainly - with the (important) exception of the semisimple radical classes - the radical classes which have been most studied are towards the supernilpotent end of the spectrum.

The present paper has three main aims:

(1) to present some examples of (hereditary) subidempotent radical classes;

(2) to examine radical theory in a relatively tractable but nontrivial class of rings-the regular rings whose primitive homomorphic images are all artinian;

(3) to say something about radical classes which are closed under directs.

Investigations akin to objective (2), wherein radical theory is studied "in microcosm", may be regarded as compromise substitutes for the (probably unrealistic) aim of describing all radical classes.

The question of dirct product closure has been around for a long time. It is easy to see that a hereditary radical class with this closure property must be either supernilpotent of subidempotent, and we shall here be concerned with the latter, providing a few examples and counterexamples and answering, in the negative, Richard Wiegandt's 
question [22]: “Are hereditary, direct product-closed radical classes equationally definable?"

We shall use the following terminology and notation. A regular ring with all its primitive images artinian will be called PA-regular; $M_{n}(A)$ will denote the ring of $n \times n$ matrices over $A ; \triangleleft$ will indicate an ideal; the upper and lower radical classes defined by a class $\mathscr{A}$ will be denoted by $U(\mathscr{A})$ and $L(\mathscr{A})$ respectively; $Q$ is the field of rational numbers, $Z$ the ring of integers, $Z^{+}$the set of positive integers. All rings are associative. It never really matters, but for the sake of definiteness, "artinian" and "primitive" are to be interpreted as on the left. Finally, all classes of rings are assumed to be isomorphically closed.

1. Some radical classes of PA-regular rings. We begin by presenting some examples of radical classes consisting of PA-regular rings.

Let $\mathscr{P}$ be a class of artinian primitive rings, $\mathscr{P}^{c}$ the class of primitive rings (artinian or not) not in $\mathscr{P}$, and let $\mathscr{R}[\mathscr{P}]$ be the class of regular rings, all of whose primitive homomorphic images are in $\mathscr{P}$.

Proposition 1.1. For any class $\mathscr{P}$ of artinian primitive rings, $\mathscr{R}[\mathscr{P}]$ is the class of regular rings in $U\left(\mathscr{P}^{c}\right)$.

Proof. If $0 \neq I \triangleleft A \in \mathscr{P}^{c}$, then $I$ is primitive. If $I$ is in $\mathscr{P}$, it has an identity, and thus $A=I \oplus J$ for some ideal $J$. But then $J=0$, since $A$ is primitive, so $I=A \in \mathscr{P}^{0}$. Thus every non-zero ideal of a ring in $\mathscr{P}^{c}$ is itself in $\mathscr{P}^{c}$. It follows that $U\left(\mathscr{P}^{c}\right)$ is the class of rings without homomorphic images in $\mathscr{P}$, whence $\mathscr{R}[\mathscr{P}] \subseteq$ $U\left(\mathscr{P}^{c}\right)$. The reverse inclusion is clear.

CoRollary 1.2. For every class $\mathscr{P}$ of artinian primitve rings, $\mathscr{R}[\mathscr{P}]$ is a radical class.

COROLLARY 1.3. The class of all $\mathrm{PA}-$ regular rings is radical.

COROLlaRy 1.4. Let $\mathscr{K}$ be a class of PA-regular rings. Then $L(\mathscr{K})$ consists of $\mathrm{PA}-$ regular rings.

Some further examples, important for later work, are obtained from a consideration of the indices (of nilpotence) of nilpotent elements.

Proposition 1.5. The following conditions are equivalent for a 
regular ring $R$ and a positive integer $n$.

(i) $a^{n}=0$ for every nilpotent element a of $R$.

(ii) Every primitive homomorphic image of $R$ is isomorphic to $M_{r}(\Delta)$ for some division ring $\Delta$ and some $r \leqq n$.

(iii) $R$ is a subdirect product of rings $M_{r_{2}}\left(\Delta_{2}\right)$, where the $\Delta_{2}$ are division rings and the $r_{\lambda} \leqq n$.

Proof. (i ) $\Rightarrow$ (ii): See Kaplansky [14], Theorem 2.3.

(ii) $\Rightarrow$ (iii): $R$ is regular, and therefore semi-primitive.

(iii) $\Rightarrow$ (i ): If $b$ is a nilpotent element of $M_{r_{\lambda}}\left(\Delta_{\lambda}\right)$, where $r_{\lambda} \leqq n$ and $\Delta_{\lambda}$ is a division ring, then $b^{r_{\lambda}}=0$, so $b^{n}=0$. Thus $a^{n}=0$ for any nilpotent element $a$ of (any subring of) $\Pi M_{r_{\lambda}}\left(\Delta_{\lambda}\right)$.

From now no we shall denote by $\mathscr{R}_{n}$ the class of regular rings satisfying the conditions of Proposition 1.5.

THEOREM 1.6. For each $n, \mathscr{R}_{n}$ is a radical class which is closed under regular subrings and direct products.

Proof. If $A \in \mathscr{R}_{n}$ and $A / I \neq 0$, then since every primitive homomorphic image of $A / I$ is a homomorphic image of $A$, we have $A / I \epsilon$ $\mathscr{R}_{n}$, so $\mathscr{R}_{n}$ is homomorphically closed. If $J \triangleleft R$, and $J, R / J \in \mathscr{R}_{n}$, then certainly $R$ is regular. Both $J$ and $R / J$ are subdirect products of rings of the form $M_{r}(\Delta)$ where $\Delta$ is a division ring and $r \leqq n$. By the theorem of [20], $R$ also has such a subdirect product representation and accordingly $R$ is in $\mathscr{R}_{n}$, so the latter is closed under extensions. Finally, if $\left\{I_{\lambda} \mid \lambda \in \Lambda\right\}$ is a chain of $\mathscr{R}_{n}$-ideals of some ring, then if $a$ is a nilpotent element of $\bigcup I_{\lambda}$, there is a $\lambda_{0} \in \Lambda$ for which $a \in I_{\lambda_{0}} \in \mathscr{R}_{n}$ and thus $a^{n}=0$. Since $U I_{\lambda}$ is regular, it is in $\mathscr{R}_{n}$. Thus $\mathscr{R}_{n}$ is a radical class.

Since the defining condition on nilpotent elements is preserved by subrings and direct products and direct products of regular rings are regular, the other assertions of the theorem follow.

COROLLARY 1.7. The class of strongly regular rings is a radical class.

Proof. A regular ring is strongly regular if and only if it has no nilpotent elements (see, e.g., [6]) so the class of strongly regular rings is $\mathscr{R}_{1}$.

Corollary 1.7 was previously proved by Spircu [21] and announced by Osondu [17]. 
In a sense the examples of radical classes provided by Theorem 1.6 are special cases of those provided by Corollary 1.2: $\mathscr{R}_{n}=\mathscr{R}[\mathscr{P}]$ where $\mathscr{P}$ is the class of $r \times r$ matrix rings over division rings, $r \leqq n$. Note however that in Theorem 1.6 we require only that rings have representations as subdirect products of certain primitive rings: all primitive homomorphic images then turn out to be of this kind.

2. Lower radical representations. We turn now to the problem of finding subclasses $\mathscr{A}$, as small as possible, of radical classes $\mathscr{R}$ of PA-regular rings, such that $L(\mathscr{C})=\mathscr{R}$. It turns out that classes of matrix rings over strongly regular rings are what we seek.

THEOREM 2.1. Let $\mathscr{R}$ be a radical class consisting of PA-regular rings, and let $\mathscr{C}$ be the subclass of $\mathscr{R}$ consisting of rings isomorphic to complete matrix rings over strongly regular rings with identities. Then

(i) $\mathscr{R}=L(\mathscr{C l})$ and

(ii) $\mathscr{R}$ is hereditary.

Proof. (i) Let $R$ be a non-zero ring in $\mathscr{R}$. By Theorem 3, p. 239, of Jacobson [12], each non-zero ideal of $R$, and, in particular, $R$ itself, has an ideal $I$ isomorphic to $M_{n}(A)$ for some ring $A$ without nilpotent elements and some $n$. Furthermore, $I$ has an identity $u$ which is a central idempotent of $R$. In the proof of the cited theorem, it is established that $A \cong e R e$ for some idempotent $e \in R$. If $a \in e R e$, then $e a e=a$, while since $a$ is in $R$, there is an element $b \in R$ for which $a=a b a$. Then

$$
a(e b e) a=e a e^{2} b e^{2} a e=(e a e) b(e a e)=a b a=a .
$$

Thus $e R e$ is regular, so $A$ is. Since it has no nilpotent elements, $A$ is strongly regular. Let $\left(u_{i j}\right)$ be the identity of $M_{n}(A)$. Then for $a \in A$, we have

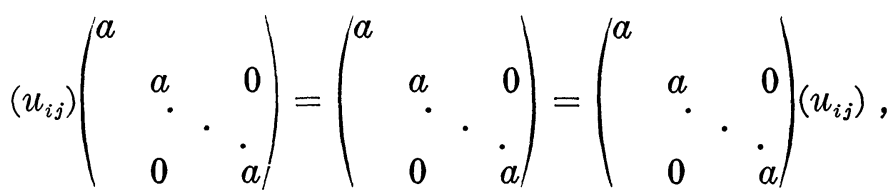

whence, comparing $(1,1)$ entries, we see that $u_{11}$ is an identity for $A$.

Since $I$ has an identity $u$, we have $R=I \oplus K$ for some $K \triangleleft R$, so, as a homomorphic image of $R, M_{n}(A) \cong I$ is in $\mathscr{R}$. This means that $I \cong M_{n}(A) \in \mathscr{M}$. Every non-zero homomorphic image of $R$ is again in $\mathscr{R}$, so it has a non-zero ideal in $\mathscr{C}$ as above. Thus $\mathscr{R}=L(\mathscr{M})$. 
(ii) Let $J \triangleleft A \in \mathscr{R}$. Then $\mathscr{R}(J) \triangleleft R$, so $J / \mathscr{R}(J) \triangleleft R / \mathscr{R}(J) \in$ $\mathscr{R}$. If $J / \mathscr{R}(J) \neq 0$, then by the theorem from [12] quoted in the proof of (i), $J / \mathscr{R}(J)$ has a non-zero ideal in $\mathscr{M} \cong \mathscr{R}$. This is impossible, so $J=\mathscr{R}(J)$, and $\mathscr{R}$ is hereditary.

COROLlaRY 2.2. Every radical class of strongly rings is hereditary.

A special case of Corollary 2.2. - all radical classes of boolean rings are hereditary - was proved in Galay's thesis [7].

It should be pointed out, however, that radical classes of regular rings need not be hereditary.

EXAMPLE 2.3. Let $R$ be the ring of all linear transformations of an $\boldsymbol{\aleph}_{0}$-dimensional vector space, $R_{0}$ the ring of linear transformations of finite rank. Then $R_{0}$ is a simple ring without identity, so $R_{0}$ has no non-zero accessible subring which is a homomorphic image of $R$. Thus $R_{0} \triangleleft R$ but $R_{0} \notin L(\{R\})$, so $L(\{R\})$ is not hereditary.

Theorem 2.1 suggests an investigation of the converse question: if $\mathscr{C}$ is a class of matrix rings over strongly regular rings (with identities), what does $L(\mathscr{C})$ look like? We shall now investigate this. The following result shows that $\mathscr{C}$ may, without loss, be assumed to be a radical class.

THEOREM 2.4. Let $\mathscr{R}$ be a radical class consisting of PA-regular rings. Then there are radical classes $\mathscr{H}_{n}, n \in Z^{+}$, consisting of strongly regular rings such that

$$
\mathscr{R}=L\left(\bigcup_{n \in Z^{+}}\left\{M_{n}(A) \mid A \in \mathscr{U}_{n}\right\}\right) .
$$

Proof. By Theorem 2.1, $\mathscr{R}=L(\overline{\mathscr{C}})$ where $\overline{\mathscr{C}}$ is the class of rings in $\mathscr{R}$ isomorphic to matrix rings over strongly regular rings (not necessarily with identities). For each $n$, let $\mathscr{U}_{n}=\{A \mid A$ is strongly regular and $\left.M_{n}(A) \in \mathscr{R}\right\}$. Since the strongly regular rings form a radical class (Corollary 1.7) and the class $\left\{A \mid M_{n}(A) \in \mathscr{R}\right\}$ is radical (see [10] or [16]), each $\mathscr{C}_{n}$ is a radical class. Clearly

$$
\mathscr{R}=L\left(\bigcup_{n \in \mathbb{Z}^{+}}\left\{M_{n}(A) \mid A \in \mathscr{\mathscr { L }}_{n}^{\prime}\right\}\right) \text {. }
$$

To finish off this section, we present some results relating the possible primitive homomorphic images of rings in a radical class $\mathscr{R}$ consisting of PA-regular rings to the classes $\mathscr{L}_{n}$ of Theorem 2.4.

Proposition 2.5. Let $N$ be a set of natural numbers, and for 
each $n \in N$ let $\mathscr{U}_{n}$ be a radical class consisting of strongly regular rings. If $R$ is in

$$
L\left(\left\{M_{n}(A) \mid n \in N, A \in \mathscr{U}_{n}\right\}\right),
$$

then $R$ is regular, and every non-zero homomorphic image of $R$ is a subdirect product of rings isomorphic to $M_{n}(\Delta), n \in N$, where $\Delta$ is a division ring in $\mathscr{U}_{n}$.

Proof. If $A \in \mathscr{U}_{n}$ and $J \triangleleft M_{n}(A)$, then $J=M_{n}(I)$ for some $I \triangleleft A$ (see, e.g., de la Rosa [19]), so $M_{n}(A) / J \cong M_{n}(A / I)$, where $A / I \in \mathscr{U}_{n}$. Thus each $\left\{M_{n}(A) \mid A \in \mathscr{U}_{n}\right\}$ is homomorphically closed. Since all rings are idempotent, every non-zero ring $R$ in $L\left(\left\{M_{n}(A) \mid n \in N, A \in \mathscr{U}_{n}\right\}\right)$ has a non-zero ideal isomorphic to $M_{n}(B)$ for some $n \in N, B \in \mathscr{U}_{n}$. By Proposition 1.6 and Corollary 1.3 (since $M_{n}(B)$ is in $\mathscr{R}_{n}$ ), $R$ is PAregular. Thus $R$ is a subdirect product of matrix rings $M_{r_{\mu}}\left(\Delta_{\mu}\right)$ over division rings $\Delta_{\mu}$. But then each such $M_{r_{\mu}}\left(\Delta_{\mu}\right)$ has a non-zero ideal isomorphic to $M_{n}(C), n \in N, C \in \mathscr{C}_{n}$. This means that $M_{r_{\mu}}\left(\Delta_{\mu}\right) \cong M_{n}(C)$, so $M_{n}(C)$, and hence also $C$, is simple. But $C$ is strongly regular, and therefore a division ring, so $r_{\mu}=n$ and $\Delta_{\mu}=C \in \mathscr{U}_{n}$ (see, e.g., Kertész [15], p. 180).

Thus $R$ is a subdirect product of matrix rings $M_{n}(\Delta), n \in N$, over division rings $\Delta \in \mathscr{U}_{n} . \quad R$ is also regular, and all the above applies equally well to any (non-zero) homomorphic image.

The next result provides an alternative formulation of the previous one (cf. Proposition 1.5).

Proposition 2.6. The following conditions are equivalent for a regular ring $R$ and a class $\mathscr{P}$ of matrix rings of bounded order over division rings.

(i) Every primitive homomorphic image of $R$ is in $\mathscr{P}$.

(ii) Every homomorphic image of $R$ is a subdirect product of rings in $\mathscr{P}$.

Proof. ( i ) $\Rightarrow$ (ii): Each homomorphic image $R / I$ of $R$ is regular, and thus a subdirect product of primitive rings. The latter, being primitive images of $R$, are in $\mathscr{P}$.

(ii) $\Rightarrow$ (i): Let $S$ be a primitive homomorphic image of $R$. Then $S$ is a subdirect product of rings in $\mathscr{P}$, so for every nilpotent element $a$ of $S$ we have $a^{n}=0$, where $n$ is an upper bound for the orders of the matrix rings in $\mathscr{P}$. This forces $S$ to be simple artinian, and hence in $\mathscr{P}$ (see Proposition 1.5).

CoRollary 2.7. Let $N$ be a set of natural numbers, and for 
each $n \in N$ let $\mathscr{Q}_{n}$ be a radical class consisting of strongly regular rings. If $R \in L\left(\left\{M_{n}(A) \mid n \in N, A \in \mathscr{U}_{n}\right\}\right)$ then every primitive homomorphic image of $R$ is isomorphic to a matrix ring $M_{n}(\Delta)$, where $n \in N$ and $\Delta$ is a division ring in $\mathscr{C}_{n}$.

3. Radical theory for strongly regular rings. In a strongly regular ring, each element $a$ has associated with it a unique element $a^{\prime}$ such that

$$
a=a^{2} a^{\prime}=a a^{\prime} a=a^{\prime} a^{2} \text { and } a^{\prime}=\left(a^{\prime}\right)^{2} a=a^{\prime} a a^{\prime}=a\left(a^{\prime}\right)^{2} .
$$

Thus the strongly regular rings are equipped with an extra unary operation, ', and if this, as well as the ring operations, is taken into account, then the class of strongly regular rings is a variety.

This variety can serve as a universal class for radical theory. Since as a class of rings, the class of strongly regular rings is hereditary and homomorphically closed, it can serve as a universal class of rings also. Moreover, the notions of homomorphism, kernel and image are the same whichever way one looks at things, so we get the same radical classes either way, and can accordingly afford to blur the distinction.

Now when a hereditary radical class $\mathscr{C}$ of rings is used as a universal class, every radical class in $\mathscr{U}$ is a radical class of rings. This was first noted (for topological rings) by Arnautov and Vodincear [2]; for a proof, see also [10]. Thus radical classes of strongly regular rings (in any sense) are radical classes of rings. Moreover, in view of the representation in Theorem 2.4, they assume a crucial rôle in the wider context of radical theory for PA-regular rings.

Varieties of regular rings (where account is taken of ' as well as the rings operations) provide examples.

THEOREM 3.1. Let or be a variety of strongly regular rings. Then

(i) $\mathscr{Y}$ is generated by the division rings it contains, and

(ii) $\mathscr{V}$ is closed under extensions.

Proof. (i) Every $A \in \mathscr{V}$ is a subdirect product (qua ring and $q u a$ strongly regular ring) of division rings, and the latter are necessarily in $\mathscr{V}$.

(ii) Let $A$ be a strongly regular ring with an ideal $I$ such that $I$ and $A / I$ are in $\mathscr{Y}$. Then $A$ has a family $\left\{J_{\lambda} \mid \lambda \in \Lambda\right\}$ of ideals such that each $A / J_{\lambda}$ is a division ring and $\bigcap_{A} J_{\lambda}=0$. If, for some $\lambda$, $I \subseteq J_{\lambda}$, then $A / J_{\lambda}$, as a homomorphic image of $A / I$, is in $\mathscr{T}$, while if $I \nsubseteq J_{\lambda}$, then by maximality, $I+J_{\lambda}=A$, so $A / J_{\lambda} \cong I / I \cap J_{\lambda} \in \mathscr{V}$. Hence $A$ is in $\mathscr{Y}$. 
COROLLARY 3.2. Every variety of strongly regular rings is a radical class.

\section{Proof. As for Theorem 1.4 of [8].}

One can obtain examples of varieties of strongly rings by intersecting the class of strongly regular rings with varieties of rings. There are others, however. For instance, whereas the variety of rings generated by $Q$ is the class of commutative rings, the variety of strongly regular rings generated by $Q$ consists of rings with characteristic 0 and thus excludes, for example, all finite fields. Another non-trivial example of a variety is the class of all strongly regular rings of characteristic 0 .

A variety of strongly regular rings is generated by its subclass of division rings; it's also defined by a set of identities which involve ' as well as the ring operations. But for division rings, we have $a^{\prime}=a^{-1}$ if $a \neq 0$ while $0^{\prime}=0$. Thus, for example, the variety of strongly regular rings of characteristic 0 is defined by the set of identities

$$
\left\{p a^{2}(p a)^{\prime}=a \mid p \text { prime }\right\} .
$$

The structure of the set of idempotents provides further examples of radical classes of strongly regular rings. If $A$ is strongly regular, then all idempotents in $A$ are central, and the set $E(A)$ of all idempotents is a boolean ring with respect to the multiplication of $A$ and the new addition given by

$$
e+f=e+f-2 e f .
$$

THEOREM 3.3. Let $\mathscr{R}$ be a radical class of boolean rings, $\mathscr{R}^{*}$ the class of strongly regular rings $A$ for which $E(A) \in \mathscr{R}$. Then $\mathscr{R}^{*}$ is a radical class.

Proof. If $A \in \mathscr{R}^{*}$ and $I \triangleleft A$, then for every $e \in E(A / I)$ there is an $\bar{e} \in E(A)$ such that $\bar{e}+I=e$ (see [11], p. 52: Lallement's Lemma). The natural homomorphism from $A$ to $A / I$ therefore induces a surjective homomorphism from $E(A)$ to $E(A / I)$. Accordingly, $E(A / I) \in$ $\mathscr{R}$, so that $A / I \in \mathscr{R}^{*}$ and it follows that $\mathscr{R}^{*}$ is homomorphically closed.

Now let $B$ be a strongly regular ring with an ideal $J$ such that $J$ and $B / J$ are in $\mathscr{R}^{*}$. The natural map $B \rightarrow B / J$ induces a surjective homomorphism $E(B) \rightarrow E(B / J)$, with kernel $\{e \in E(B) \cdot \mid e \in J\}=E(J)$. Since $E(J)$ and $E(B / J)$ are in $\mathscr{R}$, so is $E(B)$, i.e., $B$ is in $\mathscr{R}^{*}$, and the latter is closed under extensions. 
If $\left\{L_{\lambda} \mid \lambda \in \Lambda\right\}$ is a chain of $\mathscr{R}^{*}$-ideals of a strongly regular ring $R$ and $R=\bigcup_{\lambda} L_{\lambda}$, then $E(R)=\bigcup_{\lambda} E\left(L_{\lambda}\right) \in \mathscr{R}$, since $E\left(L_{\lambda}\right) \triangleleft E(R)$ for each $\lambda$, so $R \in \mathscr{R}^{*}$. This completes the proof.

Some non-obvious examples of radical class of boolean rings can be obtained as follows from some results of Cramer [5]. For each cardinal number $\alpha$, let $\mathscr{F}_{\alpha}$ be the class of boolean rings $A$ such that for $a \in A$, the principal ideal of $A$ generated by $a$ has, when viewed as a boolean algebra, no free subalgebras of rank $\alpha$. Details of this and other examples are given in Galay's thesis [7].

4. Radical classes obtained from varieties. We return to the question of the production of radical classes consisting of PA-regular rings from radical classes of strongly regular rings. In this section we shall look at the case of varieties of strongly regular rings from this viewpoint, and direct some attention to the problem of deciding when the resultant radical classes of PA-regular rings are closed under direct products.

THEOREM 4.1. Let $\mathscr{U}$ be a variety of regular rings, $N$ a set of positive integers. Then a ring $R$ is in $L\left(\left\{M_{n}(A) \mid A \in \mathscr{Q}\right\}\right)$ if and only if $R$ is regular and every primitive homomorphic image of $R$ is isomorphic to $M_{n}(\Delta)$ for some division ring $\Delta$ in $\mathscr{Z}$.

Proof. "Only if" follows from Corollary 2.7. (By Corollary 3.2, $\mathscr{U}$ is a radical class.)

For the converse, let $R$ be a regular ring for which every primitive homomorphic image is isomorphic to $M_{n}(\Delta), n \in N, \Delta \in \mathscr{U}$. Then $R$ is PA-regular, so by the proof of Theorem 2.1, $R$ has a non-zero ideal $L \cong M_{r}(Y)$, for some $r$, where $Y$ is a strongly regular ring with identity. Then $L$ is a homomorphic image of $R$, so all of its primitive images are isomorphic to rings $M_{n}(\Delta), n \in N, \Delta \in \mathscr{U}$. Let $Y$ (being strongly regular) have ideals $J_{\lambda}$ such that $Y / J_{\lambda}$ is a division ring, for each $\lambda$, and $\bigcap J_{\lambda}=0$. Then for each $\lambda, M_{r}\left(Y / J_{\lambda}\right)$ is a primitive homomorphic image of $M_{r}(Y) \cong L$, so $r \in N$ and $Y / J_{\lambda} \in \mathscr{U}_{6}$ for each $\lambda$. But since $\mathscr{U}$ is closed under subdirect products, we then have $Y \in \mathscr{Q}$ and so $L$ is isomorphic to a ring in $\left\{M_{n}(A) \mid n \in N\right.$, $A \in \mathscr{U}\}$ whence $R \in L\left(\left\{M_{n}(A) \mid n \in N, A \in \mathscr{C}\right\}\right)$.

By Proposition 2.6, the last result says that for any variety $\mathscr{C}$ of strongly regular rings and set $N$ of natural numbers, $L\left(\left\{M_{n}(A) \mid A \in \mathscr{U}\right.\right.$, $n \in N\})$ is the class of regular, strongly $U\left(\left\{M_{n}(\Delta) \mid \Delta\right.\right.$ is a division ring in $\mathscr{U}, n \in N\}$ )-semi-simple rings. For (at least) some classes $\mathscr{C}$ 
of simple artinian rings, all strongly $U(\mathscr{C})$-semi-simple rings are regular. This is certainly so when there is a (ring) polynomial identity satisfied by each $R \in \mathscr{M}$, since Armendariz and Fisher [2] have shown that hereditarily idempotent rings satisfying polynomial identities are regular. From these considerations we get

COROLlaRY 4.2. Let $\mathscr{V}$ be a variety of rings, $\mathscr{U}$ the class of strongly regular rings in $\mathscr{V}, N$ a finite set of natural numbers. Then

$$
\begin{aligned}
& L\left(\left\{M_{n}(A) \mid A \in \mathscr{Q}, n \in N\right\}\right) \\
&=\{R \mid \text { Every primitive homomorphic image of } \\
& R \text { is isomorphic to a ring } M_{n}(\Delta) \text { where } n \in N \\
&\text { and } \Delta \text { is a division ring in } \mathscr{U}\} .
\end{aligned}
$$

Proof. Every ring $A$ in $\mathscr{C}$ satisfies a fixed polynomial identity $\alpha$. Let $\bar{n}=\max (N)$. Then there is an identity $\beta$ satisfied by each $M_{\bar{n}}(A)$ and therefore by each $M_{n}(A), n \in N$.

COROLlaRY 4.3. Let $\mathscr{Y}$ be a variety of rings, $N$ a finite set of natural numbers,

$$
\mathscr{C}=\left\{M_{n}(\Delta) \mid n \in N, \Delta \in \mathscr{Y}, \Delta \text { is a division ring }\right\} .
$$

Then $L\left(\left\{M_{n}(A) \mid n \in N, A \in \mathscr{Y}, A\right.\right.$ is strongly regular $\left.\}\right)$ is the class of strongly $U(\mathscr{C})$-semi-simple rings.

For the rest of this section we shall consider closure under direct products for radical classes consisting of PA-regular rings.

We do not know whether the radical class of Theorem 4.1 is closed under direct products when $N$ is finite (nor, in fact that of Corollary 4.2). However, direct product closure does require an upper bound on the indices of nilpotence of elements of rings in a radical class.

THEOREM 4.4. Let $\mathscr{R}$ be a direct product-closed radical class consisting of PA-regular rings. Then $\mathscr{R} \cong \mathscr{R}_{n}$ for some $n$.

Proof. Suppose $\mathscr{R}$ contains infinitely many rings $M_{n_{1}}\left(\Delta_{1}\right)$, $M_{n_{2}}\left(\Delta_{2}\right), \cdots$ where $\Delta_{1}, \Delta_{2}, \cdots$ are division rings and $n_{1}<n_{2}<\cdots$. Then $\Pi M_{n_{i}}\left(\Delta_{i}\right) \in \mathscr{R}$. Let $\Phi$ be a non-principal ultrafilter of subsets of $\left\{n_{1}, n_{2}, \cdots\right\}$. Then $\Pi M_{n_{i}}\left(\Delta_{i}\right) / \Phi$ is primitive (Amitsur [1], Theorem 2A) and belongs to $\mathscr{R}$. Hence $\Pi M_{n_{i}}\left(\Delta_{i}\right) / \Phi$ is isomorphic to $M_{m}(\Gamma)$ for some division ring $\Gamma$ and some $m \in Z^{+}$. But then, by Theorem $2 \mathrm{~B}$ of [1], $\left\{i \mid n_{i}=m\right\} \in \Phi$, while $\left|\left\{i \mid n_{i}=m\right\}\right|=0$ or 1 . But this can't 
happen since $\Phi$ is non-principal.

(For a discussion of ultrafilters, see, e.g. [4], Ch. VII).

The classes $\mathscr{R}_{n}$ themselves are examples of direct product closed radical classes consisting of PA-regular rings. . In [9] it was shown that for each $n$ the class $\mathscr{C}_{n}$ of hereditarily idempotent (and hence regular [2]) rings satisfying the standard identity of degree $n$ is a radical class; clearly the $\mathscr{C}_{n}$ are closed under direct products. We now present a generalization of this.

THEOREM 4.5. Let of be a variety of rings, $\mathscr{R}[\mathscr{V}]$ the class of regular rings in $\mathscr{T}$. Then $\mathscr{R}[\mathscr{Y}]$ is a hereditary radical class which is closed under direct products.

Proof. Every primitive homomorphic image of every ring in $\mathscr{R}[\mathscr{V}]$ satisfies a polynomial identity and is therefore artinian [13]. Let $M_{n}(\Delta)$ be such a ring, with $\Delta$ a division ring. Then $M_{n}(Z(\Delta))$ is in $\mathscr{T}$, so there is an upper bound on possible values of $n$. If $k$ is such an upper bound, then $\mathscr{R}[\mathscr{V}] \subseteq \mathscr{R}_{k}$, so $\mathscr{R}[\mathscr{V}] \subseteq \mathscr{R}_{k} \cap \mathscr{V}$. But $\mathscr{R}_{k} \cap \mathscr{V} \subseteq\{A \in \mathscr{V} \mid A$ is regular $\}=\mathscr{R}[\mathscr{V}]$, so $\mathscr{R}[\mathscr{V}]=\mathscr{R}_{k} \cap \mathscr{V}$. Thus $\mathscr{R}[\mathscr{V}]$ is hereditary, homomorphically closed and closed under direct products. If $\left\{I_{\lambda} \mid \lambda \in \Lambda\right\}$ is a chain of ideals of a ring $R$ and each $I_{\lambda}$ is in $\mathscr{R}[\mathscr{V}]$, then $\bigcup_{\lambda \in A} I_{\lambda}$ is regular and satisfies all the identities of the $I_{\lambda}$, so it is in $\mathscr{V}$. All that is now needed is to prove that $\mathscr{R}[\mathscr{\mathscr { V }}]$ is closed under extensions. If $I \triangleleft T$ and if $I, T / I \in \mathscr{R}[\mathscr{V}]$, then certainly $T$ is regular. Each of $I, T / I$ is a subdirect product of simple rings in $\mathscr{R}_{k} \cap \mathscr{V}$. By the theorem of Snider [20] used previously, so is $T$. This proves the theorem.

Richard Wiegandt [22] asks whether every hereditary, direct product-closed radical class is a variety. (Of course by "variety" here we mean variety of algebras possibly with other operations besides the ring operations; e.g. the Jacobson radical class is a variety when account is also taken of the circle operation and the class of strongly regular rings is a variety as we have already noted.) The answer to this question is negative. Raphael [18] notes that the class of regular rings is not a variety since it fails to be closed under equalizers; he cites the following example, attributed to Paré: $M_{2}(Q)$ has an equalizer subring which is not regular. In fact $Q$ can be replaced by any division ring $\Delta$ in this example. We now extend this to $M_{n}(\Delta)$ for all $n$.

Proposition 4.6. Let $\Delta$ be a division ring. Define $f: M_{n}(\Delta) \rightarrow$ $M_{n}(\Delta)(n>1)$ by 


$$
\begin{aligned}
& f\left(\left(a_{i j}\right)\right) \\
& \quad=\left(\begin{array}{ccc}
a_{11}-a_{12}+a_{13}-\cdots+(-1)^{n-1} a_{1 n} a_{12}-a_{13}+\cdots+(-1)^{n-2} a_{1 n} \cdots & a_{1 n} \\
\left(a_{11}+a_{21}\right)-\left(a_{12}+a_{22}\right)+\cdots+(-1)^{n-1}\left(a_{1 n}+a_{2 n}\right) & \cdots & \left(a_{1 n}+a_{2 n}\right) \\
\left(a_{21}+a_{31}\right)-\left(a_{22}+a_{32}\right)+\cdots+(-1)^{n-1}\left(a_{2 n}+a_{3 n}\right) & \cdots & \left(a_{2 n}+a_{3 n}\right) \\
\vdots & \vdots \\
\left(a_{n-1,1}+a_{n 1}\right)-\left(a_{n-1,2}+a_{n 2}\right)+\cdots+(-1)^{n-1}\left(a_{n-1, n}+a_{n n}\right) \cdots\left(a_{n-1, n}+a_{n n}\right)
\end{array}\right)
\end{aligned}
$$

Then $f$ is a ring homomorphism. Every matrix in the equalizer of $f$ and the identity has the form

$$
\left(\begin{array}{ccccc}
a & 0 & 0 & \ldots & 0 \\
* & * & * & \ldots & * \\
\vdots & \vdots & \vdots & & \vdots \\
* & * & & \ldots & \\
*
\end{array}\right) .
$$

Proof. It is clear that $f$ preserves addition. The proof of preservation of multiplication is tedious; suffice it to say that when one is calculating the $(i, j)$ entry of a product

$$
\left(\bar{a}_{i j}\right)\left(\bar{b}_{i j}\right)=f\left(\left(a_{i j}\right)\right) f\left(\left(b_{i j}\right)\right),
$$

there is a fair amount of cancelling between $\bar{a}_{i j} \bar{b}_{k j}$ and $\bar{a}_{i, k+1} \bar{b}_{k+1, j}$. If $\left(a_{i j}\right)$ is in the equalizer, then

so

$$
a_{11}=a_{11}-a_{12}+a_{13}-\cdots+(-1)^{n-1} a_{1 n},
$$

$$
\begin{aligned}
& 0=a_{12}-a_{13}+\cdots+(-1)^{n-2} a_{1 n}=a_{12}, \\
& 0=a_{13}-a_{14}+\cdots+(-1)^{n-3} a_{1 n}=a_{13} \text {, } \\
& 0=\dot{a_{1 n}} \text {. }
\end{aligned}
$$

THEOREM 4.7. Let $\mathscr{R}$ be a radical class consisting of PA-regular rings. If $\mathscr{R}$ is a variety, then $\mathscr{R}$ contains only strongly regular rings.

Proof. If $\mathscr{R}$ contains a ring which is not strongly regular, then $M_{n}(\Delta) \in \mathscr{R}$ for some division ring $\Delta$ and some $n>1$. Let $S$ be the equalizer of the identity map of $M_{n}(\Delta)$ and the map $f$ of Proposition 4.6. Let $[1]_{n 1}$ be the matrix whose $(n, 1)$ entry is 1 and whose other entries are 0 . Then $[1]_{n 1} \in S$, while for $A \in S$, we have

$$
[1]_{n 1} A[1]_{n 1}=[1]_{n 1}\left(\begin{array}{ccccc}
a & 0 & 0 & \ldots & 0 \\
* & * & * & \ldots & * \\
\vdots & \vdots & \vdots & & \vdots \\
* & * & * & \ldots & *
\end{array}\right)[1]_{n 1}=\left(\begin{array}{ccccc}
0 & 0 & 0 & \cdots & 0 \\
0 & 0 & 0 & \ldots & 0 \\
\vdots & \vdots & \vdots & & \vdots \\
a & 0 & 0 & \ldots & 0
\end{array}\right)[1]_{n 1}=0
$$


Thus there is no $A \in S$ for which $[1]_{n 1} A[1]_{n 1}=[1]_{n 1}$, whence $S$ is not regular, so certainly $S \notin \mathscr{R}$. But if $\mathscr{R}$ were a variety, it would be closed under equalizers.

\section{REFERENCES}

1. S. A. Amitsur, Prime rings having polynomial identities with arbitrary coefficients, Proc. London Math. Soc., (3) 17 (1967), 470-486.

2. E. P. Armendariz and J. W. Fisher, Regular P.I. rings, Proc. Amer. Math. Soc., 39 (1973), 247-251.

3. В.И. Арнаутов и М.И. Водинчар [V.I. Arnautov and M.I. Vodincear], Радикальь mопологических колеч [Radicals of topological rings], Mat. Issled. 3 vyp. 2(8) (1968), 31-61.

4. D. W. Barnes and J.M. Mack, An Algebraic Introduction to Mathematical Logic, New York-Heidelberg-Berlin, Springer, 1975.

5. T. Cramer, Extensions of free boolean algebras, J, London Math. Soc., (2) 8 (1974), 226-230.

6. A. Forsythe and N. H. McCoy, On the commutativity of certain rings, Bull. Amer. Math. Soc., 52 (1946), 523-526.

7. T. A. Galay, Radical Classes of Boolean Algebras, Ph. D. Thesis, University of British Columbia, Vancouver, 1974.

8. B. J. Gardner, Semi-simple radical classes of algebras and attainability of identities, Pacific J. Math. 61 (1975), 401-416.

9. - Polynomial identities and radicals, Compositio Math., 35 (1977), 269-279.

10. B. J. Gardner and P. N. Stewart, Reflected radical classes, Acta Math. Acad. Sci. Hungar, 28 (1976), 293-298.

11. J. M. Howie, An Introduction to Semigroup Theory, London-New York-San Francisco, Academic Press, 1976.

12. N. Jacobson, Structure of Rings, Amer. Math. Soc. Colloquium Publs. vol. XXXVII (rev. ed.) Providence, 1964.

13. I. Kaplansky, Rings with a polynomial identity, Bull. Amer. Math. Soc., 54 (1948), $575-580$.

14. - Topo'ogical representation of algebras. II, Trans. Amer. Math. Soc., 68 (1950), 62-75.

15. A. Kertész, Vorlesungen über Artinsche Ringe, Budapest, Akadémiai Kiado, 1968.

16. J. Krempa, Radicals of semi-group rings, Fund. Math., 85 (1974), 57-71.

17. K. E. Osondu, Von Neumann regular rings with unique inverses, Notices Amer. Math. Soc., 23 (1976), Abstract No. 731-16-2, p. A-70.

18. R. Raphael, Some remarks on regular and strongly regular rings, Canad. Math. Bull., 17 (1974/75), 709-712.

19. B. de la Rosa, $A$ radical class which is fully determined by a lattice isomorphism, Acta. Sci. Math. (Szeged) 33 (1972), 337-341,

20. R. L. Snider, Subdirect decompositions of extension rings, Michigan Math. J., 16 (1969), 225-226.

21. T. Spircu, Radicalul strict regulat al unui inel, Stud. Cerc. Mat., 26 (1974), 751-754.

22. R. Wiegandt, Radical and Semisimple Classes of rings, Kingston, Ontario, Queen's University, 1974.

Received January 9, 1980.

UNIVERSITY OF TASMANIA

Hobart, Australia 



\title{
PACIFIC JOURNAL OF MATHEMATICS
}

\section{EDITORS}

\author{
DONALD BABBITT (Managing Editor) \\ University of California \\ Los Angeles, CA 90024 \\ Hugo RossI \\ University of Utah \\ Salt Lake City, UT 84112 \\ C. C. MOORE and Arthur Agus \\ University of California \\ Berkeley, CA 94720
}

\section{J. DugundjI}

Department of Mathematics

University of Southern California

Los Angeles, CA 90007

R. FinN and J. Milgram

Stanford University

Stanford, CA 94305

\section{ASSOCIATE EDITORS}
R. ARENS
E. F. BeCKENBACH
B. H. NeumanN
F. WOLF
K. YoshidA

\section{SUPPORTING INSTITUTIONS}

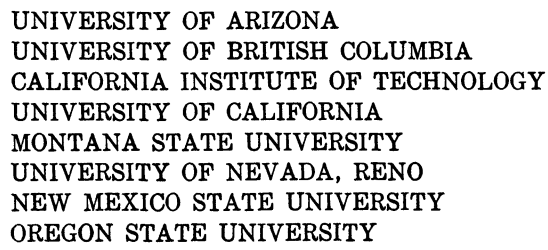

UNIVERSITY OF ARIZONA

UNIVERSITY OF BRITISH COLUMBIA

CALIFORNIA INSTITUTE OF TECHNOLOGY

UNIVERSITY OF CALIFORNIA

MONTANA STATE UNIVERSITY

UNIVERSITY OF NEVADA, RENO

NEW MEXICO STATE UNIVERSITY

OREGON STATE UNIVERSITY

\author{
UNIVERSITY OF OREGON \\ UNIVERSITY OF SOUTHERN CALIFORNIA \\ STANFORD UNIVERSITY \\ UNIVERSITY OF HAWAII \\ UNIVERSITY OF TOKYO \\ UNIVERSITY OF UTAH \\ WASHINGTON STATE UNIVERSITY \\ UNIVERSITY OF WASHINGTON
}

The Supporting Institutions listed above contribute to the cost of publication of this Journal, but they are not owners or publishers and have no responsibility for its content or policies.

Mathematical papers intended for publication in the Pacific Journal of Mathematics should be in typed form or offset-reproduced, (not dittoed), double spaced with large margins. Please do not use built up fractions in the text of the manuscript. However, you may use them in the displayed equations. Underline Greek letters in red, German in green, and script in blue. The first paragraph or two must be capable of being used separately as a synopsis of the entire paper. Please propose a heading for the odd numbered pages of less than 35 characters. Manuscripts, in triplicate, may be sent to any one of the editors. Please classify according to the scheme of Math. Reviews, Index to Vol. 39. Supply name and address of author to whom proofs should be sent. All other communications should be addressed to the managing editor, or Elaine Barth, University of California, Los Angeles, California, 90024.

50 reprints to each author are provided free for each article, only if page charges have been substantially paid. Additional copies may be obtained at cost in multiples of 50 .

The Pacific Journal of Mathematics is issued monthly as of January 1966. Regular subscription rate: $\$ 102.00$ a year (6 Vols., 12 issues). Special rate: $\$ 51.00$ a year to individual members of supporting institutions.

Subscriptions, orders for numbers issued in the last three calendar years, and changes of address shoud be sent to Pacific Journal of Mathematics, P.O. Box 969, Carmel Valley, CA 93924, U.S.A. Old back numbers obtainable from Kraus Periodicals Co., Route 100, Millwood, NY 10546.

\section{PUBLISHED BY PACIFIC JOURNAL OF MATHEMATICS, A NON-PROFIT CORPORATION}

Printed at Kokusai Bunken Insatsusha (International Academic Printing Co., Ltd.). 8-8, 3-chome, Takadanobaba, Shinjuku-ku, Tokyo 160, Japan. 


\section{Pacific Journal of Mathematics}

Vol. 99, No. $2 \quad$ June, 1982

Thomas E. Armstrong and Karel Libor Prikry, On the semimetric on a

Boolean algebra induced by a finitely additive probability measure . . . .249

Walter Russell Bloom, Strict local inclusion results between spaces of

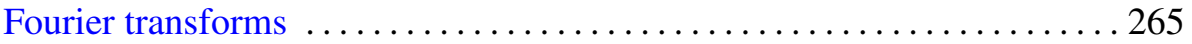

Richard Clark Brown, Notes on generalized boundary value problems in

Banach spaces. II. Infinite-dimensional extension theory ........... 271

Sui Sun Cheng, Isoperimetric eigenvalue problem of even order differential

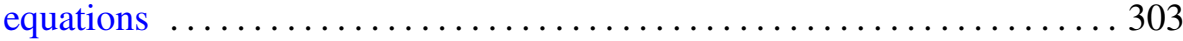

Lung O. Chung and Jiang Luh, Derivations of higher order and

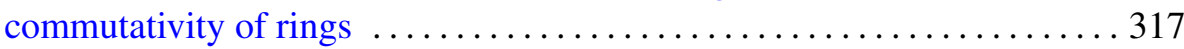

Ali Ahmad Fora, A fixed point theorem for product spaces . . . . . . . . . 327

Barry J. Gardner, Radical classes of regular rings with Artinian primitive

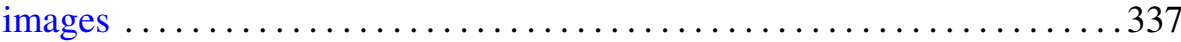

John Brady Garnett and Peter Wilcox Jones, BMO from dyadic BMO . . . 351

Allen E. Hatcher, On the boundary curves of incompressible surfaces . . . . 373

Richard Howard Hudson and Kenneth S. Williams, Resolution of

ambiguities in the evaluation of cubic and quartic Jacobsthal sums .....379

Viktor Losert, Counter-examples to some conjectures about doubly

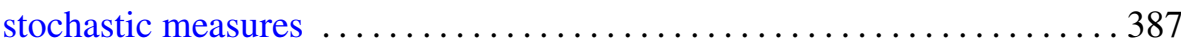

Kenneth Derwood Magill, Jr., P. R. Misra and Udai Bhan Tewari,

Structure spaces for sandwich semigroups

Mark Mandelker, Continuity of monotone functions

Kenneth Guy Miller, An index theorem and hypoellipticity on nilpotent Lie groups ......................................... 419

Evelyn M. Nelson, Homomorphisms of mono-unary algebras . . . . . . . . . 427

Marvin E. Ortel, The support of an extremal dilatation . . .

R. S. Pathak and O. P. Singh, Finite Hankel transforms of distributions . . . 439

Richard Cole Penney, The theory of ad-associative Lie algebras

Linda Ruth Sons, Zero distribution of functions with slow or moderate

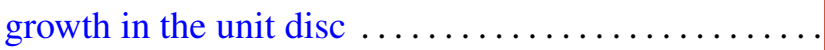

Russell Bruce Walker, Transversals to laminations 